\section{Nickelallergien: ausgezeichneter Forscher}

\author{
Dr. Marc Schmidt vom Universitätsklinikum Gießen ist Preisträger \\ des diesjährigen ADF/ECARF-Award. Er wurde ausgezeichnet für \\ seine Forschungsarbeiten zur Pathogenese und zur Therapie von \\ Nickelallergien.
}

$\mathrm{s}$ eit dem Jahr 2005 vergibt die Arbeitsgemeinschaft Dermatologische Forschung (ADF) zusammen mit der Europäischen Stiftung für Allergieforschung (ECARF) den ADF/ECARF-Award. Den mit 5.000 Euro dotieren Preis erhielt dieses Jahr am 18. Februar in Tübingen ein Team des Zentrums für Dermatologie, Venerologie und Allergologie des Universitätsklinikums Gießen unter Leitung des Biologen Dr. Marc Schmidt. Mit ihrem Projekt „Ausschlaggebende Rolle des menschlichen Toll-like-Rezeptors 4 bei der Entstehung von Nickelallergien" konnte die Arbeitsgruppe grundlegende neue Erkenntnisse zur Pathogenese der Nickelallergie präsentieren.

„Der Preis ist mir sehr wichtig, denn die Resultate unserer Forschung machen Hoffnung, dass Nickelallergiker zukünftig mit neuen, spezifischen Medikamenten behandelt werden können, was bisher nicht möglich war", erklärte Schmidt. Es war bereits bekannt, dass sowohl ein spezifisches Signal von T-Lymphozyten als auch ein entzündlicher Stimulus notwendig sind, um eine Nickelallergie auszulösen. Wie dieses entzündliche Signal beschaffen ist, konnte die Arbeitsgruppe um Schmidt jetzt enthüllen. Demnach aktivieren $\mathrm{Ni}^{2+}$-Ionen direkt den Tolllike-Rezeptor 4 (TLR 4) und steuern so die die proinflammatorische Genexpression. Genauere Einzelheiten legt der Preisträger gemeinsam mit Prof. Dr. Matthias Goebeler in dieser Ausgabe des Allergo Journal in seinem Beitrag „Neues zur Pathophysiologie des allergischen Kontaktekzems auf Nickel“ auf den Seiten 74-80 dar. In weiteren Untersuchungen will die Forschergruppe nun herausfinden, wie die spezifische NickelAndockstelle des TLR-4 mithilfe von Medikamenten blockiert werden kann, ohne dass gleichzeitig die Immunabwehr negativ beeinflusst wird.

Dr. Marc Schmidt wurde 1971 geboren und studierte in Würzburg Lebensmittelchemie, später Biologie. Von 1997 bis 2001 forschte er für seine Promotion am Institut für Medizinische Strahlenkunde und Zellforschung der Universität Würzburg. Seit Februar 2005 ist Schmidt Forschungsgruppenleiter, zunächst an der Klinik für Dermatologie, Allergologie und Venerologie des Universitätsklinikums Mannheim und seit Februar 2010 an der gleichnamigen Abteilung des Universitätsklinikums Gießen.

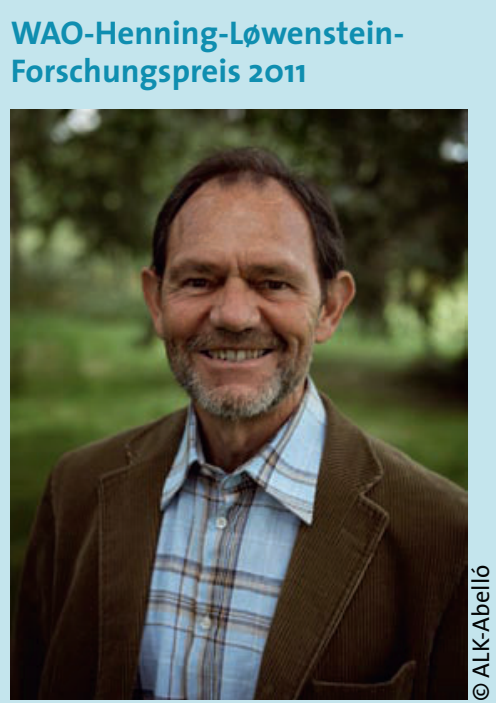

Henning Løwenstein

Das Pharmaunternehmen ALK-Abelló und die World Allergy Organization WAO loben auch dieses Jahr wieder gemeinsam den WAO-HenningLøwenstein-Forschungspreis aus. Mit dem Preis sollen ein Anreiz für ein besonderes Forschungsengagement geschaffen und herausragende wissenschaftliche Leistungen im Bereich der Allergologie honoriert werden. Er ist mit 20.000 Euro dotiert und wird zusammen mit einem Reisestipendium zum World Allergy Congress in Cancún, Mexiko, im Dezember 2011 vergeben.

Der Henning-Løwenstein-Forschungspreis der WAO ist nach Henning Løwenstein benannt. Der dänische Pionier der Allergieforschung hatte in den $70 e r$ Jahren wesentlichen Anteil an der bahnbrechenden Entdeckung neuer Methoden zur Allergenstandardisierung. Løwenstein war ehemaliger Executive Vice President Research \& Development bei ALKAbelló.

Die Bewerbungsunterlagen sind in englischer Sprache an untenstehende Anschrift zu senden. Weitere Informationen sind unter www.alk-abello.com/ research/rdatalk/researchaward/pages/ home.aspx online abrufbar. Einsendeschluss ist der 30. Juni 2011.

The WAO Henning Løwenstein Award 2011

ALK A/S Boge Alle 1 2970 Horsholm Denmark 